\title{
Randomised, double-blind, placebo-controlled trial of fructo-oligosaccharides in active Crohn's disease
}

\author{
Jane L Benjamin, ${ }^{1}$ Charlotte R H Hedin, ${ }^{1}$ Andreas Koutsoumpas, ${ }^{1}$ Siew C Ng, ${ }^{2,3}$ \\ Neil E McCarthy, ${ }^{4}$ Ailsa L Hart, ${ }^{2}$ Michael A Kamm, ${ }^{2,5}$ Jeremy D Sanderson, ${ }^{1}$ \\ Stella C Knight, ${ }^{2}$ Alastair Forbes, ${ }^{6}$ Andrew J Stagg, ${ }^{4}$ Kevin Whelan, ${ }^{1}$ \\ James 0 Lindsay ${ }^{4,7}$
}

\section{See Commentary, p 882}

- Additional materials are published online only. To view these files please visit the journal online (http://gut.bmj. com).

${ }^{1}$ Nutritional Sciences Division, King's College London, London, UK

${ }^{2}$ Antigen Presenting Research Group, Imperial College, London, UK

${ }^{3}$ Institute of Digestive Disease Chinese University of Hong Kong, Hong Kong

${ }^{4}$ Blizard Institute of Cell and

Molecular Science, Barts and the London School of Medicine and Dentistry, Queen Mary University of London, London, UK

${ }^{5}$ Departments of Medicine and Gastroenterology, St Vincent's Hospital, University of Melbourne, Melbourne, Australia

${ }^{6}$ Centre for Gastroenterology and Nutrition, University College, London, UK

${ }^{7}$ Digestive Diseases Clinical Academic Unit, Barts and the London NHS Trust, London, UK

\section{Correspondence to} Dr James Lindsay, Digestive Diseases Clinical Academic Unit, Barts and the London School of Medicine, The Royal London Hospital, London E1 1BB, UK; james.lindsay@

bartsandthelondon.nhs.uk

For further details of protocol see http://www.

controlled-trials.

com/ISRCTN50422530.

Accepted 6 December 2010 Published Online First 24 January 2011

\section{ABSTRACT}

Introduction The commensal intestinal microbiota drive the inflammation associated with Crohn's disease. However, bacteria such as bifidobacteria and Faecalibacterium prausnitzii appear to be immunoregulatory. In healthy subjects the intestinal microbiota are influenced by prebiotic carbohydrates such as fructo-oligosaccharides (FOS). Preliminary data suggest that FOS increase faecal bifidobacteria, induce immunoregulatory dendritic cell (DC) responses and reduce disease activity in patients with Crohn's disease. Aims and methods To assess the impact of FOS in patients with active Crohn's disease using an adequately powered randomised double-blind placebo-controlled trial with predefined clinical, microbiological and immunological end points. Patients with active Crohn's disease were randomised to $15 \mathrm{~g} /$ day FOS or nonprebiotic placebo for 4 weeks. The primary end point was clinical response at week 4 (fall in Crohn's Disease Activity Index of $\geq 70$ points) in the intention-to-treat (ITT) population.

Results 103 patients were randomised to receive FOS $(n=54)$ or placebo $(n=49)$. More patients receiving FOS (14 $(26 \%)$ vs $4(8 \%) ; p=0.018)$ withdrew before the 4-week end point. There was no significant difference in the number of patients achieving a clinical response between the FOS and placebo groups in the ITT analysis (12 (22\%) vs 19 (39\%), $p=0.067)$. Patients receiving FOS had reduced proportions of interleukin (IL)-6-positive lamina propria DC and increased DC staining of IL-10 $(p<0.05)$ but no change in IL-12p40 production. There were no significant differences in the faecal concentration of bifidobacteria and $F$ prausnitzii between the groups at baseline or after the 4-week intervention. Conclusion An adequately powered placebo-controlled trial of FOS showed no clinical benefit in patients with active Crohn's disease, despite impacting on DC function. ISRCTN50422530.

\section{INTRODUCTION}

Crohn's disease (CD) is a chronic inflammatory condition of the gastrointestinal tract driven by abnormal $\mathrm{T}$ cell responses to the intestinal microbiota. However, the commensal microbiota also shape the development of the intestinal immune system and protect against inflammation in animal models. ${ }^{1-3}$ Specific bacterial species have distinct immunological effects mediated by dendritic cells (DC) which sample bacteria from the intestinal lumen and direct the subsequent functional

\section{Significance of this study}

What is already known about this subject?

- Active Crohn's disease is associated with alterations in intestinal microbiota (dysbiosis).

- Bifidobacteria and Faecalibacterium prausnitzii enhance intestinal dendritic cell immunoregulatory responses and are reduced in active disease.

- Fructo-oligosaccharides increase faecal bifidobacteria and $F$ prausnitzii in healthy subjects.

- An open-label trial in patients with mildly active Crohn's disease found that fructo-oligosaccharides increased faecal bifidobacteria, increased intestinal dendritic cell interleukin 10 release and reduced disease activity.

\section{What are the new findings?}

- Fructo-oligosaccharides do not enhance bifidobacteria and $F$ prausnitzii in patients with active Crohn's disease.

- This treatment was shown to be ineffective at inducing remission in an adequately powered double-blind placebo-controlled trial.

- Fructo-oligosaccharides do impact on intestinal dendritic cell function.

How might it impact on clinical practice in the foreseeable future?

- Fructo-oligosaccharides are not an effective treatment for active Crohn's disease.

- Further studies of the microbiological, immunological and clinical effects of fructo-oligosaccharides in maintaining disease remission are required.

- A clinical benefit of an intervention that exerts a prebiotic effect cannot be excluded.

differentiation of naive $\mathrm{T}$ cells into effector or regulatory populations. ${ }^{4} 5$ Studies on human DC have demonstrated immunoregulatory effects of bifidobacteria, which increase DC interleukin (IL)-10 production and decrease interferon- $\gamma$ release from DC-activated allogeneic CD4+T cells. ${ }^{6}$ Likewise, the firmicute Faecalibacterium prausnitzii reduces peripheral blood mononuclear cell IL-12 and increases IL-10 release in vitro. ${ }^{7}$

In support of the differential effects of bacteria on intestinal inflammation, marked differences in 
both faecal and mucosal microbiota (dysbiosis) in patients with active CD compared with both inactive disease and healthy subjects have been reported. ${ }^{3}$ Although results vary owing to differences in both the patient groups studied and the microbiological method used, higher concentrations of bacteroides and Escherichia coli ${ }^{8}{ }^{9}$ and lower concentrations of bifidobacteria ${ }^{10}$ and $F$ prausnitzii ${ }^{71}$ have been reported in faecal and mucosal samples from patients with CD compared with healthy controls. Lower concentrations of $F$ prausnitzii have been found in patients with active disease than in those with quiescent disease, and low levels of this organism in CD resection specimens predict subsequent endoscopic disease recurrence. ${ }^{7} 11$

The importance of the intestinal microbiota in the aetiology of mucosal inflammation provides the rationale for therapeutic strategies using probiotics and prebiotics in patients with CD. Despite clear evidence of benefit in animal models, well-designed studies of probiotics have shown no benefit in either the treatment of active disease or the prevention of clinical and endoscopic recurrence after ileocaecal resection. ${ }^{12-15}$ Prebiotics are non-digestible selectively fermented short-chain carbohydrates that stimulate the growth and/or activity of a limited number of the gut microbiota resulting in health benefits to the host. ${ }^{16}$ For example, oligofructose and inulin are polymers of fructose found naturally in artichoke, asparagus and onions ${ }^{17}$ that increase commensal faecal and mucosal bifidobacteria and $F$ prausnitzii in healthy humans. ${ }^{18} 19$ In addition, prebiotic fermentation results in the production of short-chain fatty acids such as butyrate ${ }^{20}$ that enhance epithelial integrity and promote regulatory DC function in vitro ${ }^{21}$ In animal models of colitis, fructo-oligosaccharides (FOS) increased luminal bifidobacteria, inhibited intracellular transcription factors such as $\mathrm{NF \kappa B}$ and reduced disease activity. ${ }^{22} \mathrm{~A}$ pilot open-label study investigating the effect of $15 \mathrm{~g}$ /day FOS in mildly active CD reported a significant increase in faecal bifidobacteria, increased intestinal DC IL-10 release and a significant reduction in disease activity as assessed by the Harvey Bradshaw index. ${ }^{23}$

The aim of this study was to conduct the first randomised double-blind placebo-controlled trial with adequate power to detect the clinical, immunological and microbiological impact of FOS in a well-defined population of patients with moderately active CD.

\section{MATERIALS AND METHODS Trial design}

This is a randomised double-blind placebo-controlled trial conducted in line with CONSORT recommendations. Patients were recruited from four inflammatory bowel disease outpatient clinics in London. Based on the results of our previous open-label study $^{23}$ and assuming a response rate of $50 \%$ in the intervention group and $20 \%$ in the placebo group, a total of 51 patients would be required in each group to detect a difference between interventions with a significance level of 0.05 and a power of $90 \%$.

\section{Patients}

Adult patients with a documented diagnosis of $\mathrm{CD}$ for at least 3 months and a Crohn's Disease Activity Index (CDAI) $\geq 220$ with one additional marker of inflammation (elevated $\mathrm{C}$ reactive protein (CRP)/erythrocyte sedimentation rate/platelet count) were included. Exclusion criteria included the use of anti-tumour necrosis factor agents in the preceding 12 weeks; antibiotics, probiotics or prebiotics in the preceding 4 weeks; rectal preparations during the preceding 2 weeks; and any non-steroidal anti-inflammatory drugs during the preceding week. Further exclusion criteria included a change in dose of immunosuppressant within 12 weeks and oral 5-aminosalicylic acid or steroids within 4 weeks. The maximum permissible steroid dose was $20 \mathrm{mg} /$ day. Other exclusion criteria included short bowel syndrome, previous pan-proctocolectomy/significant colonic resection, sepsis, pure perianal disease and positive stool culture for an enteropathogen.

\section{Trial protocol}

Informed consent was obtained prior to any study-related procedures. During the 1 -week screening patients completed a diary to calculate disease activity (CDAI) and gave baseline bloods with genotyping of common CARD 15 mutations. Eligible patients were recruited and a fresh faecal sample was collected for molecular microbiology and faecal calprotectin analysis. Rectal biopsies were taken from normal rectal mucosa at an unprepared flexible sigmoidoscopy for DC immunological analysis from a subset of patients recruited at St Mark's Hospital. Patients completed the Inflammatory Bowel Disease Questionnaire (IBDO, McMaster University, Canada). They were given a symptom diary to monitor disease activity and side effects.

A random number sequence was generated independently to designate treatment assignment. Patients were randomised 1:1 to each group stratified by disease localisation (pure colonic disease vs ileal involvement) and hospital site. Sachets of FOS and placebo were packed in identical sequentially numbered envelopes according to this code at each site. Both patients and researcher were blinded to treatment assignation. Patients randomised to the intervention group supplemented their normal diet with $15 \mathrm{~g} /$ day FOS (Synergy1, Beneo-Orafti, Belgium) which is classified as a food supplement by the UK Medicines and Healthcare Products Regulatory Agency. This comprised fructose polymers of differing chain lengths $(70 \%$ oligofructose with a degree of polymerisation (DP) $<10$ and $30 \%$ inulin with a DP $>10)$. Lower DP oligofructose is more rapidly fermented in the proximal colon than higher DP inulin. Patients in the placebo group received $15 \mathrm{~g}$ /day of a non-prebiotic carbohydrate (maltodextrin). Both FOS and maltodextrin were provided as identical $7.5 \mathrm{~g}$ sachets and patients were advised to consume two sachets per day mixed with food or a cold beverage for 4 weeks. Unused sachets were collected at the end of the study period to calculate adherence. All patients remained on an unrestricted diet and stable maintenance therapy for their CD throughout the trial.

After the 4-week intervention period, patients were assessed by the same investigator who remained blinded to treatment allocation. The baseline investigations were repeated. Symptom diaries were interrogated and rated on a scale of 0 (absent), 1 (mild), 2 (moderate), 3 (severe) and a sum of the daily scores was calculated. ${ }^{24}$ Patients experiencing a significant adverse event or deterioration in disease activity requiring medical intervention were withdrawn from the trial and treated with standard therapy for $\mathrm{CD}$. All randomised patients were included in the intentionto-treat (ITT) population and the 4-week safety analysis.

\section{Clinical end points}

The primary end point was defined as the percentage of patients achieving a clinical response (fall in CDAI of $\geq 70$ points) in the ITT population at week 4 . Predefined secondary end points included the percentage of patients achieving disease remission (CDAI $\leq 150$ points) at week 4 , clinical response and remission in the per protocol group, as well as a change in CDAI, CRP, faecal calprotectin and quality of life (IBDQ) at week 4 . There 
were no changes to the protocol or trial end points after recruitment commenced.

\section{Cytokine production by intestinal DCs}

Ongoing DC cytokine production in the absence of exogenous stimulation was assessed by intracellular staining and multicolour flow cytometry of antibody-labelled dissociated lamina propria mononuclear cells (LPMC) harvested from rectal biopsies as previously described (full methodology given in online supplement). ${ }^{25}$ For intracellular cytokine staining, paired cultures of LPMC with and without monensin were compared. Cells were surface-labelled with anti-HLA-DR (BD Bioscience, Oxford, UK), anti-CD11c (Dako, Ely, UK) and DC exclusion cocktail (lin; AbD Serotec, Oxford, UK), then fixed and permeabilised with leucoperm A and B. Anti-cytokine antibody (anti-IL-10-PE (AbD Serotec), anti-IL-12p40-PE (BD Pharmingen, New Jersey, USA and R\&D Systems, Minneapolis, USA) and anti-IL-6-PE (R\&D Systems) were added. Data were acquired using a FACSCalibur flow cytometer (Beckton Dickinson, Oxford, UK). Using multicolour analysis, DC were identified as an HLA-DR+ lineage (CD3-, CD14-, CD16-, CD19-, CD34-) population, and within this gate, the $\mathrm{CD} 11 \mathrm{c}+$ (conventional DC) population was assessed. The proportion of cytokine-positive cells was determined by SED normalised subtraction using WinList software (Verity Software House). The proportion of cytokineproducing cells and the level of staining (intensity ratio) in cytokine-positive cells were determined.

\section{Faecal bifidobacteria and Faecalibacterium prausnitzii}

Faecal bifidobacteria and $F$ prausnitzii were quantified in fresh faecal samples using fluorescent in situ hybridisation as previously described (full methodology given in online supplement). ${ }^{23}$ Total cell counts were quantified using the nucleic acid stain 4,6-diamidino-2-phenlindole. Cells were hybridised with Cy3-labelled oligonucleotide probes targeting regions of $16 \mathrm{~S}$ rRNA specific for bifidobacteria (5'-GCT GCC TCC CGT AGG AGT- $\left.3^{\prime}\right)^{26}$ and $F$ prausnitzii (5'-CCT CTG CAC TAC TCA AGA AAA AC- $\left.3^{\prime}\right)^{27}$ (Microsynth, Balgach, Switzerland). The hybridised bacteria were quantified manually by fluorescence microscopy and concentrations expressed as cells/g dry faeces.

\section{Faecal calprotectin}

The CALPRO Calprotectin ELISA test kit (Nova Tec, Dietzenbach, Germany) was used according to the manufacturer's instructions.

\section{Statistical analysis}

Statistical analyses were performed using SPSS software package V.16 (SPSS Software). Data were tested for normality using the Kolmogrov-Smirnov and Shapiro-Wilk tests. The results are shown as mean (SD). The clinical outcome variables were assessed by ITT and per protocol analyses. Comparison of categorical data between patients in the FOS and placebo groups was performed using the $\chi^{2}$ method. If any cell in these analyses had an expected count of $<5$, the Fisher exact test was used. Comparison of continuous data between FOS and placebo was performed using the Student $t$ test. Comparison of continuous data within groups was performed using paired $t$ tests. The results for all tests were considered significant if $p<0.05$.

\section{RESULTS}

\section{Patient cohort}

Recruitment took place between September 2006 and April 2009 when complete accrual was achieved. The final trial visit was in
May 2009. Of 217 patients screened, 95 did not meet the inclusion criteria 56 had normal inflammatory markers, 33 had a CDAI $<220,1$ had a CDAI $>450,3$ changed medication, 1 had a positive stool culture and 1 was diagnosed with hepatitis $C$ ) and 19 declined to participate. A total of 103 subjects were therefore randomised (54 to FOS, 49 to placebo) and comprise the ITT population (consort diagram available in online supplement). Mean (SD) disease duration was 11 (7.7) years in the FOS group and 10 (7.6) years in the placebo group $(p=0.48)$; there were no significant demographic differences between groups (table 1). A single mutation in the CARD 15 gene was present in $16(16.3 \%)$ patients with one patient having a compound heterozygote genotype (R702W and G908R), with no difference in prevalence between groups.

\section{Clinical outcomes}

In the ITT population there was no significant difference in the number of patients achieving the primary end point of clinical response at week 4 between patients in the FOS group ( $n=12$, $22 \%)$ and the placebo group ( $n=19,39 \% ; p=0.067)$. Likewise, there was no significant difference in the numbers achieving clinical remission between groups (FOS $\mathrm{n}=6,11 \%$ vs placebo $\mathrm{n}=10,20 \%, \mathrm{p}=0.193$ ). The lack of difference in response and remission rates remained when analysed per protocol (table 2). At week 4 there was no significant difference in the mean CDAI between the FOS and placebo groups (mean (SD) 250 (84.9) vs 220 (88.7); $p=0.112$ ). Predefined subgroup analysis revealed no benefit of FOS in patients with isolated colonic disease or ileal involvement. There were no significant differences in IBDO scores between groups (per protocol) at baseline (FOS 120.3 (30.0) vs placebo 129.8 (26.5); $p=0.122$ ), whereas at week 4 the FOS group had a significantly lower IBDO score than the placebo group (129.9 (36.3) vs 149.8 (24.5); $\mathrm{p}=0.004$ ).

In the per protocol group there were no differences in the mean (SD) CRP level between the FOS and placebo groups at baseline (18.8 (21.6) $\mathrm{mg} / \mathrm{l}$ vs $20.8(23.1) \mathrm{mg} / \mathrm{l} ; \mathrm{p}=0.692)$ or at 4 weeks (20.9 (26.4) mg/l vs 20.2 (24.1) mg/l; p=0.902). Faecal calprotectin concentrations were available at baseline and at 4 weeks in 60 patients (28 FOS, 32 placebo). There were no significant differences in the mean (SD) values between groups at either baseline (FOS $621.4(559.4) \mathrm{mg} / \mathrm{kg}$ vs placebo 647.9 (533.3) $\mathrm{mg} / \mathrm{kg}$; $\mathrm{p}=0.852$ ) or week 4 (FOS 657.4 (592.5) mg/kg vs

Table 1 Characteristics of patients in the fructo-oligosaccharide (FOS) and placebo groups

\begin{tabular}{lccl}
\hline & FOS (n=54) & Placebo (n=49) & p Value \\
\hline Male, n (\%) & $18(33 \%)$ & $22(45 \%)$ & 0.229 \\
Mean (SD) age, years & $40(14.8)$ & $39(13.7)$ & 0.633 \\
Mean (SD) duration of CD, years & $11(7.7)$ & $10(7.6)$ & 0.477 \\
Disease site, n (IC/colonic/SB) & $39 / 10 / 5$ & $33 / 12 / 4$ & 0.759 \\
Resection, n (\%) & $24(44 \%)$ & $14(29 \%)$ & 0.111 \\
Smoker, n (\%) & $18(33 \%)$ & $11(22 \%)$ & 0.220 \\
Mean (SD) CDAl at randomisation & $283(61)$ & $286(62)$ & 0.794 \\
Mean (SD) IBDO at randomisation & $121(31)$ & $129(27)$ & 0.169 \\
Medication & & & \\
$\quad$ Immunosuppression, n (\%) & $24(44 \%)$ & $26(53 \%)$ & 0.382 \\
Azathioprine, n (\%) & $19(35 \%)$ & $20(41 \%)$ & 0.556 \\
Methotrexate, n (\%) & $4(7 \%)$ & $6(12 \%)$ & 0.408 \\
5-Aminosalicylates, n (\%) & $22(41 \%)$ & $25(51 \%)$ & 0.296 \\
Prednisolone, n (\%) & $3(6 \%)$ & $3(6 \%)$ & 0.902 \\
\hline
\end{tabular}

$\mathrm{p}$ Values calculated by the Student $\mathrm{t}$ test for continuous data and $\chi^{2}$ test or Fisher exact test for categorical data.

$C D$, Crohn's disease; CDAI, Crohn's Disease Activity Index; IBDQ, Inflammatory Bowel Disease Questionnaire; IC, ileocolonic; SB, small bowel. 
Table 2 Response and remission rates in the fructo-oligosaccharide (FOS) and placebo groups

\begin{tabular}{|c|c|c|c|}
\hline & FOS & Placebo & p Value \\
\hline \multicolumn{4}{|l|}{ Response $^{*}$} \\
\hline Intention to treat, $\mathrm{n}(\%)$ & $12(22 \%)$ & $19(39 \%)$ & 0.067 \\
\hline Per protocol, n (\%) & $12(30 \%)$ & $19(42 \%)$ & 0.243 \\
\hline \multicolumn{4}{|l|}{ Remission $\dagger$} \\
\hline Intention to treat, $\mathrm{n}(\%)$ & $6(11 \%)$ & $10(20 \%)$ & 0.193 \\
\hline Per protocol, n (\%) & $6(15 \%)$ & $10(22 \%)$ & 0.395 \\
\hline
\end{tabular}

placebo 829.8 (635.9) $\mathrm{mg} / \mathrm{kg}, \mathrm{p}=0.322$ ). However, there was a smaller increase in faecal calprotectin during the trial in the FOS group (mean (SD) $+61.5(300.6) \mathrm{mg} / \mathrm{kg}$ ) compared with the placebo group (mean (SD) +296 (493.6) mg/kg; $\mathrm{p}=0.092$ ).

\section{Adherence, gastrointestinal tolerance and adverse events}

All patients who completed the intervention showed good adherence with a mean (SD) intake of 89 (18.7)\% of sachets in the FOS group and 92 (11.8)\% of sachets in the placebo group $(\mathrm{p}=0.355)$. The intake in those who completed the study was 13.3 (2.8) g/day in the FOS group compared with 13.8 (1.8) g/ day in the placebo group ( $p=0.355)$.

At baseline there were no differences in the incidence or severity of gastrointestinal symptoms between groups. During week 4 there was a higher incidence of borborygmi in the FOS group (4.7 (2.5) days/week) than in the placebo group (4.0 (2.6) days/week, $p=0.013$ ). However, no differences were seen in the incidence of any other symptoms. Throughout the trial, patients in the FOS group had a significantly greater severity of flatulence $(p=0.004)$, borborygmi $(p=0.029)$ and abdominal pain $(p=0.048)$ than those in the placebo group (table 3$)$.

Significantly more patients in the FOS group than in the placebo group withdrew before the end of the trial $(n=14,26 \%$ vs $n=4,8 \% ; p=0.0018)$. Of those who withdrew, 10 patients $(19 \%)$ in the FOS group did so because of worsening symptoms compared with $3(6 \%)$ in the placebo group $(p=0.059)$. Baseline calprotectin levels (when available) were significantly higher in

Table 3 Comparison of severity of symptoms between fructooligosaccharide (FOS) and placebo groups at baseline and during the 4-week trial period

\begin{tabular}{lcccc}
\hline Severity & Baseline $^{*}$ & p Value & Trial score $\dagger$ & p Value \\
\hline Acid reflux & & & & \\
$\quad$ FOS & $2.5(3.6)$ & & $4.0(2.8)$ & \\
$\quad$ Placebo & $3.0(3.9)$ & 0.487 & $2.8(3.5)$ & 0.218 \\
Rumbling gut & & & & \\
$\quad$ FOS & $7.7(4.9)$ & & $8.3(4.0)$ & \\
$\quad$ Placebo & $7.8(5.3)$ & 0.900 & $6.0(6.9)$ & 0.029 \\
Abdominal bloating & & & & \\
$\quad$ FOS & $8.8(5.5)$ & & $9.6(5.5)$ & \\
$\quad$ Placebo & $7.7(5.7)$ & 0.321 & $7.4(4.6)$ & 0.061 \\
Abdominal pain & & & $10.2(5.2)$ & \\
$\quad$ FOS & $11.2(4.8)$ & & $8.0(2.7)$ & 0.048 \\
$\quad$ Placebo & $10.8(4.3)$ & 0.667 & $10.8(5.2)$ & \\
Flatulence & & & $8.0(4.7)$ & 0.004 \\
$\quad$ FOS & $8.3(5.0)$ & & 0.780 & \\
$\quad$ Placebo & $8.0(5.5)$ & &
\end{tabular}

*Mean (SD) cumulative score over a 7-day period using the following scoring system: $0=$ absent; $1=$ mild; $2=$ moderate; $3=$ severe.

† Mean cumulative average score for the 4-week trial period. those patients receiving FOS who subsequently withdrew due to worsening symptoms $(n=5 ; 1068.5(594.9) \mathrm{mg} / \mathrm{kg})$ than in those who completed the trial ( $\mathrm{n}=23 ; 524.1$ (514.2) $\mathrm{mg} / \mathrm{kg}$; $\mathrm{p}=0.046)$. There were no differences in other baseline characteristics between patients who withdrew and those who did not (data not shown). Serious adverse events were reported in four patients receiving FOS (three related to disease deterioration, one admitted for an acute asthma attack) and two patients receiving placebo (one disease deterioration and one car accident). No other adverse events were recorded.

\section{Immunological analysis}

Mucosal biopsies from histologically non-inflamed rectum at both time points were available from 26 patients (13 patients randomised to each group). In keeping with our previous publication, ${ }^{23}$ there was a significant increase in DC IL-10 staining between baseline and week 4 in patients in the FOS group (median (IOR) intensity ratio increased from $1.3(0.6)$ to 2.0 (1.6); $p=0.035$ ), whereas no difference was seen in patients receiving placebo (intensity ratio at baseline and week 4 was 1.4 (1.4) and 1.6 (1.0); $p=0.655$, figure 1 ). There were no significant differences in IL-12p40 production by intestinal DC (percentage positive or intensity ratio) between groups at baseline or week 4 (not shown). IL-6 production by DC is a key factor in overcoming the inhibitory activity of $\mathrm{T}$ regulatory cells and enabling the development of effector responses. ${ }^{28}$ There was a significant reduction in the mean (SD) proportion of IL-6-positive DC during the intervention in patients receiving FOS (from 29 (12) to $-32(15) ; \mathrm{p}=0.036)$ compared with placebo (from 32 (18) to -28 (15); $\mathrm{p}=0.464$, figure 2 ).

\section{Microbiological analysis}

There were no significant differences in faecal concentrations of bifidobacteria or F prausnitzii between the groups at baseline. In contrast to studies of healthy volunteers, there was no significant difference in faecal concentrations of bifidobacteria at week

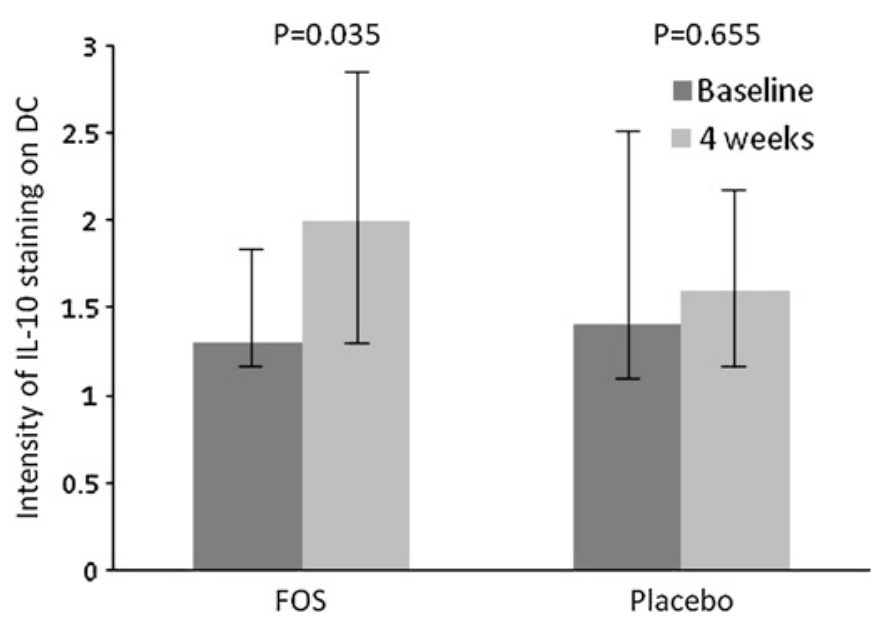

Figure 1 Lamina propria mononuclear cells were dissociated from mucosal biopsies taken from non-inflamed rectum at baseline and week $4(n=13$ per group). Dendritic cells (DC) were identified as an HLA-DR+ lineage-(CD3-, CD14-, CD16-, CD19-, CD34-) population and, within this gate, the $\mathrm{CD} 11 \mathrm{c}+$ population was assessed. The intensity of interleukin 10 (IL-10) expression was determined after intracellular cytokine staining using SED normalised subtraction. Staining of cells cultured in the absence of monensin was subtracted from staining of cells cultured in the presence of monensin, giving a measure of ongoing cytokine production. The median (IOR) intensity of CD11c+ DC IL-10 staining at baseline and week 4 is shown. FOS, fructo-oligosaccharide. 
Proportion of IL-6 + DC at baseline and week 4

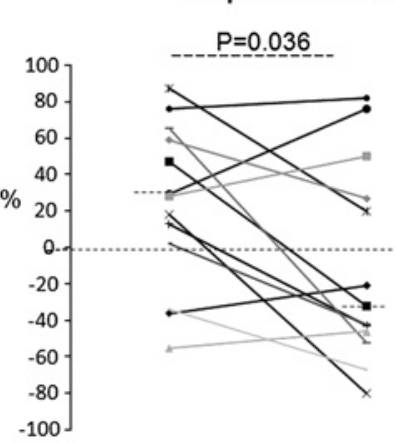

FOS

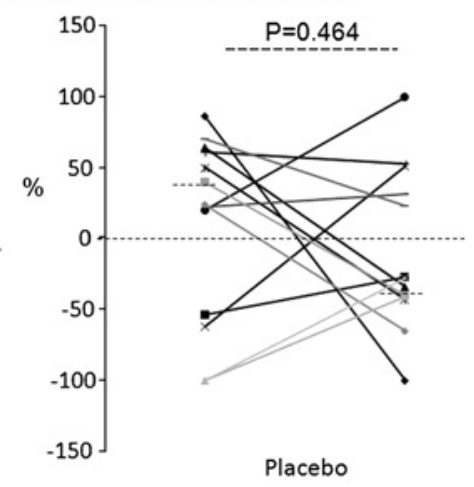

Placebo
Figure 2 Lamina propria mononuclear cells were dissociated from mucosal biopsies taken from non-inflamed rectum at baseline and week 4 ( $n=13$ per group). Dendritic cells (DC) were identified as an HLA-DR+ lineage-(CD3-, CD14-, CD16-, CD19-, CD34-) population and, within this gate, the CD11c+ population was assessed. The proportion of interleukin 6 (IL-6)-positive cells was determined after intracellular cytokine staining using SED normalised subtraction. Staining of cells cultured in the absence of monensin was subtracted from staining of cells cultured in the presence of monensin, giving a measure of ongoing cytokine production. The percentage of IL-6-positive $\mathrm{CD} 11 \mathrm{c}+\mathrm{DC}$ for each patient at baseline and week 4 is shown. FOS, fructo-oligosaccharide.

4 between patients in the FOS and placebo groups (9.4 (0.6) $\log _{10}$ cells/g dry faeces vs 9.3 (0.8) $\log _{10}$ cells/g dry faeces; $\mathrm{p}=0.201$ ). In addition, there was no significant difference in faecal concentrations of $F$ prausnitzii between patients in the FOS and placebo groups at week 4 (8.7 (0.9) $\log _{10}$ cells/g dry faeces vs 8.7 (1.0) $\log _{10}$ cells/g dry faeces; $p=0.95$ ).

In order to investigate the absence of a prebiotic-induced bifidogenesis in patients receiving FOS, the baseline characteristics of patients who did experience a rise in bifidobacteria were compared with those who did not. Patients receiving FOS who experienced a large increase $\left(>+0.5 \log _{10}\right.$ cells $\left./ g\right)$ in bifidobacteria $(n=7)$ had a lower CDAI at baseline than patients with no increase in bifidobacteria $((n=32) 248.1$ (30.0) vs 290.0 (56.8); $\mathrm{p}=0.064)$. Likewise, they had lower faecal calprotectin concentrations (324.0 (468.1) mg/kg vs $683.2(545.5) \mathrm{mg} / \mathrm{kg} ; \mathrm{p}=0.130)$. Previous ileocolic resection did not correlate with change in bifidobacteria. Finally, as previously reported, ${ }^{29}$ an inverse correlation was found between the baseline concentration of bifidobacteria and the change in concentration of bifidobacteria following FOS (figure 3).

There was no significant difference in the CDAI change between baseline and 4 weeks in patients receiving FOS who did experience bifidogenesis compared with those that did not $(-29$ (88.2) vs -35 (79.6); $p=0.855)$. There was also no significant difference in CRP between those with a bifidogenic effect and those without, either at baseline (24.8 (23.8) vs 16.4 (21.2), $\mathrm{p}=0.311)$ or at 4 weeks (25.5 (27.0) vs 18.6 (25.7), $\mathrm{p}=0.508)$.

\section{DISCUSSION}

We report the first randomised double-blind placebo-controlled trial of prebiotics in patients with active CD. The trial was adequately powered to detect a clinically relevant difference in response rates between patient groups and achieved complete recruitment. The power calculation was based upon results from our pilot open-label study and the placebo rate from a published meta-analysis. ${ }^{30}$ In order to ensure participants had symptoms related to active inflammation and to minimise placebo response

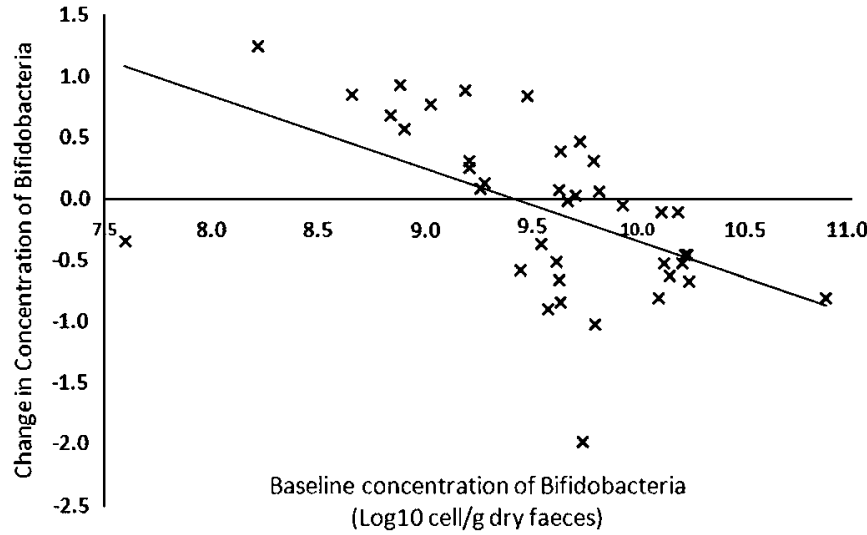

Figure 3 Correlation between baseline concentrations of faecal bifidobacteria ( $\log _{10}$ cells/g dry faeces) and their change in concentration after $15 \mathrm{~g} /$ day fructo-oligosaccharide (FOS) for 4 weeks in patients randomised to the FOS group in whom stool samples were available at both time points $(n=40 ; r=-0.618, p<0.001)$.

rates, inclusion required a CDAI consistent with moderately active disease as well as another marker of active inflammation. We found no significant difference in the number of patients meeting the primary end point (fall in CDAI of $\geq 70$ points) between the FOS and placebo groups. Likewise, there was no difference between groups in the number of patients achieving clinical remission $(\mathrm{CDAI} \leq 150)$ or any other clinical secondary end point.

FOS was associated with a significantly increased incidence and severity of gastrointestinal symptoms and more patients randomised to this group withdrew from the trial than from the placebo group. Gastrointestinal symptoms relating to FOS in patients with inflammatory bowel disease probably result from both an osmotic effect and bacterial fermentation. ${ }^{31} 32$ However, this is unlikely to have masked a true therapeutic benefit as per protocol analyses also showed no difference in either response or remission rates between groups. It is theoretically possible that the increase in gastrointestinal symptoms associated with FOS had a negative impact on subjective aspects of the CDAI, therefore masking a true clinical benefit. However, there were no significant improvements in objective markers of intestinal inflammation such as CRP and faecal calprotectin in patients receiving FOS compared with placebo. Finally, in view of previous reports of increased efficacy of dietary interventions in $\mathrm{CD}$ patients with ileal involvement as opposed to pure colonic disease, we stratified randomisation according to disease site. However, our prespecified subgroup analysis did not demonstrate a significant benefit of FOS compared with placebo in either patients with ileal involvement or those with disease limited to the colon, although the trial may be underpowered to detect a difference in this subgroup analysis. We therefore conclude that FOS is not an effective treatment for patients with moderately active CD.

Patients with CD often enquire about alternative non-pharmacological treatments for their disease. Indeed, we have recently reported a high prevalence of their use among patients with inflammatory bowel disease despite a lack of robust evidence demonstrating clinical benefit. ${ }^{33}$ There is therefore a clear need for randomised placebo-controlled trials to determine the efficacy of treatments that have shown promising results in open-label trials. Factors that have been reported to be associated with a high placebo response in CD trials include the number of study visits, increased study duration, low baseline 
CDAI and a normal CRP. ${ }^{30}$ Despite designing the protocol to minimise the placebo rate, there was a high clinical response rate in patients receiving placebo. Future studies of nutritional interventions in this patient group will need to modify the power calculation to take account of this.

Prebiotic carbohydrates such as FOS are selectively fermented in the colon resulting in beneficial alterations in the commensal microbiota and short-chain fatty acid production. ${ }^{18-20} \mathrm{~A}$ number of studies in healthy volunteers have shown that the ingestion of at least $10 \mathrm{~g} /$ day results in a marked increase in faecal and mucosal bifidobacteria and faecal $F$ prausnitzii. ${ }^{18-20}$ In the context of mildly active $\mathrm{CD}$, our previous open-label trial showed that $15 \mathrm{~g} /$ day FOS resulted in a significant increase in faecal bifidobacteria. ${ }^{23}$ Surprisingly, in this placebo-controlled trial patients given $15 \mathrm{~g} /$ day FOS did not experience significant changes in faecal bifidobacteria. The documented adherence of 13.3 (2.8) g/day was sufficient to induce bifidogenesis. ${ }^{34}$ Analysis of the demographic features of the patients receiving FOS who experienced the greatest bifidogenesis showed that disease duration, previous ileocaecal resection and medication did not impact on the microbiological response to FOS. However, there was a significant negative correlation between baseline bifidobacteria concentration and subsequent increase after FOS (an observation previously observed in healthy subjects ${ }^{29}$ ), which suggests that patients with $\mathrm{CD}$ who have the lowest baseline levels had the greatest potential for bifidogenesis. Interestingly, the baseline bifidobacteria concentrations in patients randomised to the FOS group in the current trial were significantly higher than those reported in our previous open-label trial where bifidogenesis did occur (9.5 (0.5) vs 8.8 (0.9) $\log _{10}$ cell/g; $\left.p=0.024\right)$. Patients recruited to this open-label trial had less severe disease at study entry than those recruited to the current trial. ${ }^{23}$ In keeping with this, patients experiencing an increase in bifidobacteria in our current trial had less active disease with lower baseline CDAI score and faecal calprotectin levels. We therefore suggest that prebiotic carbohydrates may have less impact on the intestinal microbiota in patients with significant intestinal inflammation, although there was no significant difference in the clinical response of patients receiving FOS who did experience an increase in bifidobacteria compared with those who did not.

Recent evidence suggests that modulation of DC function contributes to the proinflammatory and immunoregulatory effects of the intestinal microbiota on the mucosal immune system. ${ }^{35}$ Thus, successful treatment with the probiotic VSL\#3 in patients with ulcerative colitis is associated with a reduction in DC IL-12p40 and an increase in DC IL-10. ${ }^{36}$ In our previous open-label study in patients with mildly active CD, $15 \mathrm{~g} /$ day FOS increased the percentage of intestinal DC expressing IL-10. ${ }^{23}$ We hypothesised that this effect was dependent on the bifidogenic effect of FOS acting via Toll-like receptors or other pattern recognition receptors. However, despite no effect on the faecal microbiota in the current trial, FOS still resulted in significant alterations in cytokine production by intestinal DC compared with placebo, with an increase in IL-10 staining and a reduction in IL- 6 production. These data indicate that the changes in DC cytokine expression are unlikely to result from alterations in intestinal bifidobacteria. Alternative explanations include shortchain fatty acid production following colonic fermentation of the prebiotic as FOS has been reported to increase faecal butyrate production in an in vitro fermentation system. ${ }^{37}$ Butyrate influences DC maturation and cytokine production in vitro, altering the balance of IL-12 and IL-10 production in favour of the latter. ${ }^{21}$ In our current study, increased production of IL-10 and reduced IL- 6 by DC indicated that DC cytokine production was skewed by FOS towards a less inflammatory profile, but this was evidently not sufficient to impact upon clinical disease. Strikingly, unlike VSL\#3, FOS did not alter DC IL-12p40 release, which suggests that alterations in IL-12 or IL-23 production may be more clinically relevant. It remains to be tested whether the effects on DC may be significant in the context of maintenance of remission or disease prevention.

In conclusion, an adequately powered trial of FOS in a welldefined population of patients with active CD showed no clinical benefit despite impacting on DC immunology. A higher severity of gastrointestinal symptoms was seen in patients receiving FOS, and patient withdrawal was greater in this group. This may add support to the concept that fermentable carbohydrates may worsen functional symptoms associated with CD. ${ }^{38}$ In contrast to previous studies in healthy subjects and patients with mild $\mathrm{CD}$, no significant alteration in faecal microbiota was seen in this cohort of patients with moderately active $\mathrm{CD}$. Our findings suggest that future research regarding prebiotics in inflammatory bowel disease should focus on their role in maintaining disease remission or perhaps preventing its onset in those with a high-risk phenotype.

\section{Acknowledgements We are grateful to the following consultant}

gastroenterologists for facilitating recruitment of patients from their Inflammatory Bowel Disease Clinics: Professor Rampton (Barts and the London NHS Trust); Drs Bloom, Langmead and McCartney (University College Hospitals Foundation Trust): Drs Gabe, Nightingale and Pitcher (St Mark's Hospital). We are also grateful for the help and support provided by the dedicated research nurses Belinda Theis (UCH) and Jenny Burch (St Mark's Hospital). Investigators at University College London had the support of the National Institute of Health Research (NIHR) via the Comprehensive Biomedical Research Centre at this site. Finally we would like to acknowledge and thank the patients who took part in this study. Without their time and effort, this study would not have been possible.

Funding This work was supported by a research grant from the Broad Medical Research Program, Los Angeles, California, USA. Sachets of fructo-oligosaccharides and placebo were provided by Beneo-Orafti, Belgium.

\section{Competing interests None.}

Ethics approval This study was conducted with the approval of the National Research Ethics Service (South East Research Ethics Committee).

Contributors JLB: patient recruitment, molecular microbiology, data analysis, drafted manuscript, statistical analysis. CRHH: study design, patient recruitment, molecular microbiology. AK: patient recruitment, molecular microbiology. SCN: patient recruitment, immunology, data analysis. NEMcC: immunology, data analysis, statistical analysis. ALH: principal investigator St Mark's, immunology, critical revision of manuscript with important intellectual content. MAK: principal investigator St Mark's, immunology, critical revision of manuscript with important intellectual content. JDS: principal investigator Guy's and St Thomas', critical revision of manuscript with important intellectual content. SCK: supervised immunological analysis, critical revision of manuscript with important intellectual content, study concept and trial design. AF: principal investigator UCLH, data analysis, study concept and trial design critical revision of manuscript with important intellectual content. AJS: trial concept and study design, supervised immunological analysis, critical revision of manuscript with important intellectual content. KW: trial concept and study design, supervised molecular microbiology, data analysis, statistical analysis, drafted and revised manuscript. JOL: chief investigator for clinical trial, study concept and trial design, supervised all clinical recruitment, data analysis, drafted and revised manuscript.

Provenance and peer review Not commissioned; externally peer reviewed.

\section{REFERENCES}

1. Ivanov II, Frutos RL, Manel N, et al. Specific microbiota direct the differentiation of IL-17-producing T-helper cells in the mucosa of the small intestine. Cell Host Microbe 2008:4:337-49.

2. Rakoff-Nahoum S, Paglino J, Eslami-Varzaneh F, et al. Recognition of commensal microflora by toll-like receptors is required for intestinal homeostasis. Cell 2004;118:229-41.

3. Sartor RB. Microbial influences in inflammatory bowel diseases. Gastroenterology 2008;134:577-94.

4. Rescigno M, Urbano M, Valzasina B, et al. Dendritic cells express tight junction proteins and penetrate gut epithelial monolayers to sample bacteria. Nat Immunol $2001 ; 2: 361-7$ 
5. Stagg AJ, Hart AL, Knight SC, et al. The dendritic cell: its role in intestinal inflammation and relationship with gut bacteria. Gut 2003;52:1522-9.

6. Hart AL, Lammers K, Brigidi P, et al. Modulation of human dendritic cell phenotype and function by probiotic bacteria. Gut 2004:53:1602-9.

7. Sokol H, Pigneur $B$, Watterlot $L$, et al. Faecalibacterium prausnitzii is an antiinflammatory commensal bacterium identified by gut microbiota analysis of Crohn disease patients. Proc Natl Acad Sci U S A 2008;105:16731-6.

8. Mylonaki M, Rayment NB, Rampton DS, et al. Molecular characterization of rectal mucosa-associated bacterial flora in inflammatory bowel disease. Inflamm Bowel Dis 2005;11:481-7.

9. Swidsinski A, Ladhoff A, Pernthaler A, et al. Mucosal flora in inflammatory bowel disease. Gastroenterology 2002;122:44-54.

10. Tamboli CP, Neut C, Desreumaux P, et al. Dysbiosis in inflammatory bowel disease. Gut 2004;53:1-4.

11. Sokol H, Seksik P, Furet JP, et al. Low counts of Faecalibacterium prausnitzii in colitis microbiota. Inflamm Bowel Dis 2009;15:1183-9.

12. Marteau P, Lemann M, Seksik P, et al. Ineffectiveness of Lactobacillus johnsonii LA1 for prophylaxis of postoperative recurrence in Crohn's disease: a randomised, double blind, placebo controlled GETAID trial. Gut 2006;55:842-7.

13. Rolfe VE, Fortun PJ, Hawkey CJ, et al. Probiotics for maintenance of remission in Crohn's disease. Cochrane Database Syst Rev 2006;(4):CD004826.

14. Hedin C, Whelan $\mathrm{K}$, Lindsay JO. Evidence for the use of probiotics and prebiotics in inflammatory bowel disease: a review of clinical trials. Proc Nutr Soc 2007:66:307-15.

15. Marteau $\mathbf{P}$, Sokol H, Dray X, et al. Bacteriotherapy for inflammatory bowel disease: therapeutic tool and/or pharmacological vectors? Gastroenterol Clin Biol 2009;33: S228-34

16. Leenen $\mathbf{C H}$, Dieleman LA. Inulin and oligofructose in chronic inflammatory bowel disease. J Nutr 2007;137:2572S-5S.

17. van Loo $L$, Coussement $P$, de Leenheer $L$, et al. On the presence of inulin and oligofructose as natural ingredients in the western diet. Crit Rev Food Sci Nutr 1995;35:525-52.

18. Gibson GR, Beatty ER, Wang $X$, et al. Selective stimulation of bifidobacteria in the human colon by oligofructose and inulin. Gastroenterology 1995;108:975-82.

19. Ramirez-Farias C, Slezak K, Fuller Z, et al. Effect of inulin on the human gut microbiota: stimulation of Bifidobacterium adolescentis and Faecalibacterium prausnitzii. Br J Nutr 2009;101:541-50.

20. Wang $\mathbf{X}$, Gibson GR. Effects of the in vitro fermentation of oligofructose and inulin by bacteria growing in the human large intestine. J Appl Bacteriol 1993:75:373-80.

21. Millard AL, Mertes PM, Ittelet D, et al. Butyrate affects differentiation, maturation and function of human monocyte-derived dendritic cells and macrophages. Clin Exp Immunol 2002;130:245-55.

22. Cherbut C, Michel C, Lecannu G. The prebiotic characteristics of fructooligosaccharides are necessary for reduction of TNBS-induced colitis in rats. J Nutr 2003:133:21-7.
23. Lindsay J0, Whelan K, Stagg AJ, et al. Clinical, microbiological, and immunologica effects of fructo-oligosaccharide in patients with Crohn's disease. Gut 2006;55:348-55

24. Pedersen A, Sandstrom B, Van Amelsvoort JM. The effect of ingestion of inulin on blood lipids and gastrointestinal symptoms in healthy females. Br J Nutr 1997:78:215-22.

25. Bell SJ, Rigby R, English $\mathrm{N}$, et al. Migration and maturation of human colonic dendritic cells. J Immunol 2001;166:4958-67.

26. Langendijk PS, Schut F, Jansen GJ, et al. Quantitative fluorescence in situ hybridization of Bifidobacterium spp. with genus-specific 16S rRNA-targeted probes and its application in fecal samples. Appl Environ Microbiol 1995;61:3069-75.

27. Suau A, Rochet V, Sghir A, et al. Fusobacterium prausnitzii and related species represent a dominant group within the human fecal flora. Syst Appl Microbiol 2001:24:139-45

28. Pasare C, Medzhitov R. Toll pathway-dependent blockade of CD4+CD25+ T cellmediated suppression by dendritic cells. Science 2003;299:1033-6.

29. Whelan K, Judd PA, Preedy VR, et al. Fructooligosaccharides and fiber partially prevent the alterations in fecal microbiota and short-chain fatty acid concentrations caused by standard enteral formula in healthy humans. J Nutr 2005;135:1896-902

30. Su C, Lichtenstein GR, Krok K, et al. A meta-analysis of the placebo rates of remission and response in clinical trials of active Crohn's disease. Gastroenterology 2004:126:1257-69

31. Gearry RB, Irving PM, Barrett JS, et al. Reduction of dietary poorly absorbed shortchain carbohydrates (FODMAPs) improves abdominal symptoms in patients with inflammatory bowel disease-a pilot study. J Crohns Colitis 2009;3:8-14.

32. Barrett JS, Gearry RB, Muir JG, et al. Dietary poorly absorbed, short-chain carbohydrates increase delivery of water and fermentable substrates to the proximal colon. Aliment Pharmacol Ther 2010:31:874-82.

33. Hedin CR, Mullard M, Sharratt E, et al. Probiotic and prebiotic use in patients with inflammatory bowel disease: a case-control study. Inflamm Bowel Dis 2010;16:2099-108.

34. Kolida S, Meyer D, Gibson GR. A double-blind placebo-controlled study to establish the bifidogenic dose of inulin in healthy humans. Eur J Clin Nutr 2007:61:1189-95.

35. $\mathbf{N g ~ S C}$, Kamm MA, Stagg AJ, et al. Intestinal dendritic cells: their role in bacteria recognition, lymphocyte homing, and intestinal inflammation. Inflamm Bowel Dis 2010;16:1787-807.

36. $\mathbf{N g} \mathbf{S C}$, Plamondon S, Kamm MA, et al. Immunosuppressive effects via human intestinal dendritic cells of probiotic bacteria and steroids in the treatment of acute ulcerative colitis. Inflamm Bowel Dis 2010;16:1286-98.

37. Probert HM, Apajalahti JH, Rautonen N, et al. Polydextrose, lactitol, and fructooligosaccharide fermentation by colonic bacteria in a three-stage continuous culture system. Appl Environ Microbiol 2004;70:4505-11.

38. Gibson PR, Shepherd SJ. Personal view: food for thought-Western lifestyle and susceptibility to Crohn's disease. The FODMAP hypothesis. Aliment Pharmacol The 2005:21:1399-409.

\section{Gut table of contents alerts}

Sign up for our electronic table of contents alert and you will never miss new issues of Gut when published online. Stay ahead and up to date by visiting gut.bmj.com. 


\section{GUT}

Randomised, double-blind, placebo-controlled trial of fructo-oligosaccharides in active Crohn's disease

Jane L Benjamin, Charlotte R H Hedin, Andreas Koutsoumpas, Siew C $\mathrm{Ng}$, Neil E McCarthy, Ailsa L Hart, Michael A Kamm, Jeremy D Sanderson, Stella C Knight, Alastair Forbes, Andrew J Stagg, Kevin Whelan and James O Lindsay

Gut 2011 60: 923-929 originally published online January 24, 2011 doi: 10.1136/gut.2010.232025

Updated information and services can be found at:

http://gut.bmj.com/content/60/7/923

\section{These include:}

Supplementary Supplementary material can be found at:

Material http://gut.bmj.com/content/suppl/2011/01/24/gut.2010.232025.DC1.ht $\mathrm{ml}$

References This article cites 37 articles, 14 of which you can access for free at: http://gut.bmj.com/content/60/7/923\#BIBL

Email alerting Receive free email alerts when new articles cite this article. Sign up in the service box at the top right corner of the online article.

Topic Articles on similar topics can be found in the following collections

Collections

Crohn's disease (909)

\section{Notes}

To request permissions go to:

http://group.bmj.com/group/rights-licensing/permissions

To order reprints go to:

http://journals.bmj.com/cgi/reprintform

To subscribe to BMJ go to:

http://group.bmj.com/subscribe/ 